\title{
ON APPLYING COMPARISON THEOREMS TO STUDYING STABILITY WITH PROBABILITY 1 OF STOCHASTIC DIFFERENTIAL EQUATIONS
}

\section{A.S. ASYLGAREEV}

\begin{abstract}
In the work we obtain two results concerning trajectory-wise properties of stochastic differential equations (SDE) with Stratonovich integral. First, we prove comparison theorems for SDE with Stratonovich integral with respect to the standard Wiener process, that is, we obtain the conditions for the coefficients of SDE, under which the solutions of one equation for a fixed trajectory of the Wiener process is always located above or below a solution to another equations for the same trajectory. At that, the drift and diffusion coefficients of the studied equations can be different. Second, on the base of the proved theorems we establish the conditions for the stability with probability 1 for perturbed solutions to scalar SDE with Stratonovich integral with respect to the trivial solution. The stability with probability 1 implies the Lyapunov stability for almost all solutions to SDE. It should be noted that, as a rule, the stability for SDE is treated in weaker sense: stability in probability, $p$-stability, exponential stability. Employing the formula of passage between Ito integral and Stratonovich integral, which is valid for sufficiently smooth coefficients of $\mathrm{SDE}$, these results can be extended to SDE with Ito integral.

The approach of the work is based on the fact that a solution to SDE can be represented as a deterministic function of a random variable solving, in its turn, a chain of ordinary differential equations with a random right hand side. Since this technique is trajectorywise, the presented results can be also reformulated for deterministic analogues of SDE, namely, for equations with symmetric integrals.
\end{abstract}

Keywords: stochastic differential equations, stability with probability 1, comparison theorems, equations with symmetric integral, Wiener process.

Mathematics Subject Classification: $60 \mathrm{H} 10$

\section{INTRODUCTION AND FORMULATION OF PROBLEM}

We consider two stochastic differential equations (SDE) with Stratonovich integral w.r.t. the standard Wiener process

$$
\begin{aligned}
& d \xi_{t}^{(1)}=\sigma_{1}\left(t, \xi_{t}^{(1)}\right) * d W_{t}+b_{1}\left(t, \xi_{t}^{(1)}\right) d t,\left.\xi_{t}^{(1)}\right|_{t=0}=\xi_{0}^{(1)}, \\
& d \xi_{t}^{(2)}=\sigma_{2}\left(t, \xi_{t}^{(2)}\right) * d W_{t}+b_{2}\left(t, \xi_{t}^{(2)}\right) d t,\left.\xi_{t}^{(2)}\right|_{t=0}=\xi_{0}^{(2)}
\end{aligned}
$$

where the functions $\sigma_{i}(t, u), b_{i}(t, u), i=1,2$, are deterministic.

The present work is a continuation of the study in [1] and it pursues two aims. The first aim is to extend the approach of [1] for comparing solutions to SDEs to a wider class of equations. To the best of our knowledge, a comparison theorem was first proved by Skorokhod [2] for Ito SDE of form $d \xi_{t}^{(i)}=b_{i}\left(t, \xi_{t}^{(i)}\right) d t+\sigma\left(t, \xi_{t}^{(i)}\right) d W(t), i=1,2$, with coinciding diffusion coefficients. Its most general form was given in monograph [3]. The method proposed by Skorokhod, was

A.S. Asylgareev, On APPLYING COMPARISON THEOREMS TO STUDYING STABILITy With PROBABILITY 1 OF STOCHASTIC DIFFERENTIAL EQUATIONS .

(C) Asylgareev A.S. 2018.

Submitted November 3, 2017. 
further developed in work [4]. Later, in [5], Geib and Mathey succeeded to extend the results by Skorokhod for Ito SDE with a multi-dimensional Wiener process and for stochastic partial differential equations (SPDE). Comparison theorems for SPDEs were also considered in work [6]. In [7], there were proved comparison theorems for Ito SDE with an additional perturbation by a Poisson process. The case of an SDE with different diffusion coefficients are less studied. The most remarkable result here is due to O'Brien, who proved comparison theorems for equations $d \xi_{t}^{(i)}=\sigma_{i}\left(\xi_{t}^{(i)}\right) d W_{t}+\frac{1}{2} \sigma_{i}\left(\xi_{t}^{(i)}\right) \sigma_{i}^{\prime}\left(\xi_{t}^{(i)}\right) d t, i=1,2$, in [8]. These equations can be rewritten in Stratonovich form as equations without drift- $d \xi_{t}^{(i)}=\sigma_{i}\left(\xi_{t}^{(i)}\right) * d W_{t}$. An essential difference of the present work is that the proven comparison theorems are applicable to an essentially wider class of SDEs than in work [8].

The second aim of the present work is to obtain the stability conditions with probability 1 for SDE

$$
\begin{gathered}
d \xi_{t}=\sigma\left(t, \xi_{t}\right) * d W(t)+b\left(t, \xi_{t}\right) d t,\left.\quad \xi_{t}\right|_{t=t_{0}}=\xi_{0}, \\
\sigma(t, 0)=0, \quad b(t, 0)=0 .
\end{gathered}
$$

Usually, the stability for SDE is considered in weaker senses: stability in probability, $p$-stability, exponential stability. We recall a perturbed solution $\xi_{t}$ to equation (3) with the initial condition $\xi_{0}=x_{0}$ is stable in probability if for each $\varepsilon>0$ it holds $\lim _{\xi_{0} \rightarrow 0} P\left\{\sup _{t>0}\left|\xi_{t}\right|>\varepsilon\right\}=0$. A perturbed solution $\xi_{t}$ to equation (3) with initial condition $\xi_{0}=x_{0}$ is $p$-stable if $\sup _{\left|x_{0}\right| \leqslant \delta, t \geqslant 0} E\left|\xi_{t}\right|^{p} \rightarrow 0$ as $\delta \rightarrow 0$.

The conditions of stability in probability and $p$-stability were given in [9], [10] and are based on constructing a Lyapunov function $V(t, x)$, for which the Lyapunov operator

$$
L=\frac{\partial}{\partial t}+\left(b(t, u)+\frac{\partial \sigma(t, u)}{\partial u} \sigma(t, u)\right) \frac{\partial}{\partial u}+\frac{1}{2}(\sigma)^{2}(t, u) \frac{\partial^{2}}{\partial u^{2}}
$$

satisfies some inequalities. Various types of exponential stability we considered in details in monograph [11].

Definition 1. A perturbed solution $\xi_{t}$ to equation (3) with initial condition $\xi_{0}=x_{0}$ is stable with probability 1 if for a.e. $\omega$, for each $\varepsilon>0$ there exists $\delta(\varepsilon, \omega)>0$ such that for each $x_{0}$ obeying $\left|x_{0}\right|<\delta$, the solution $\xi_{t}$ satisfies the inequality $\left|\xi_{t}\right|<\varepsilon$ for all $t>0$.

In the latter definition the function $\delta$ depends on $\omega$, in other works, the stability with probability 1 implies the Lyapunov stability for almost all trajectories of solutions to equation (3).

The key point of our approach is that the solutions to equations (1), (2) can be represented as $\xi_{t}^{(k)}=\varphi_{k}\left(t, W_{t}+C_{k}(t)\right), k=1,2$, where the functions $\varphi_{k}(t, u)$ are deterministic and $C_{k}(t)=$ $C_{k}(t, \omega)$ are solutions to SDE with a random right hand side (see [12]). Since this technique is in fact trajectory-wise, the obtained results can be reformulated for deterministic analogues of SDE (equations with symmetric integrals) (see [12]) and for SDEs over a wider class of processes (for instance, for SDE over a fractal Brownian motion). This study was preliminary presented on International Scientific Forum "Lomonosov-2017" [13].

\section{PRELIMINARIES}

Here we provide main ideas of monograph [12] being the base of the present work. We consider SDE with Stratonovich integral:

$$
d \xi_{t}=\sigma\left(t, \xi_{t}\right) * d W_{t}+b\left(t, \xi_{t}\right) d t .
$$

The following theorem holds for equation (4). 
Theorem 1 ([12, Ch. 2, Sect. 10, Thm. 10.1]). Let the functions $\sigma(t, u), \sigma_{t}^{\prime}(t, u), \sigma_{u}^{\prime}(t, u)$, $b(t, u)$ be continuous on $\mathbb{R}^{+} \times \mathbb{R}$. The following statements are true:

1. If the function $\xi_{t}=\varphi^{*}\left(t, W_{t}\right)$ is a solution to equation (4) such that the function $\varphi^{*}(t, u)$ possesses continuous partial derivaties $\left(\varphi^{*}\right)_{t}^{\prime}(t, u),\left(\varphi^{*}\right)_{u}^{\prime}(t, u),\left(\varphi^{*}\right)_{t u}^{\prime \prime}(t, u)$, then it satisfies the relations

$$
\left(\varphi^{*}\right)_{u}^{\prime}(t, u)=\sigma\left(t, \varphi^{*}(t, u)\right), \quad\left(\varphi^{*}\right)_{t}^{\prime}\left(t, W_{t}\right)=b\left(t, \varphi^{*}\left(t, W_{t}\right)\right) \quad \text { for all } \quad t \geqslant 0 .
$$

2. Let the function $\varphi^{*}(t, u)$ possesses continuous partial derivatives $\left(\varphi^{*}\right)_{t}^{\prime}(t, u),\left(\varphi^{*}\right)_{u}^{\prime}(t, u)$, $\left(\varphi^{*}\right)_{t u}^{\prime \prime}(t, u)$ and solves the chain of equations (5) with the initial condition $\xi_{0}=\varphi^{*}\left(0, W_{0}\right)$. Then the function $\xi_{t}=\varphi^{*}\left(t, W_{t}\right)$ is a solution to equation (4).

We introduce the notation:

$$
G(t, u)=\int_{\xi_{0}}^{u} \frac{d \psi}{\sigma(t, \psi)} .
$$

In the case, when the function $\sigma^{-1}(t, u)$ is locally summable for all $t \geqslant 0$, by the first relation in (5) the identity

$$
G(t, \varphi(t, v))=v+C(t) \quad \text { for all } \quad t \geqslant 0 \quad \text { a.s. }
$$

holds. We observe that solution (6) defines a deterministic function $\varphi(t, v)$ such that $\varphi(t, v+$ $C(t))=\varphi^{*}(t, v)$ a.s., where $C(t)=C(t, \omega)$ is a solution to an ordinary differential equation with a random right hand side

$$
C^{\prime}(t)=\frac{b\left(t, \varphi\left(t, W_{t}+C(t)\right)\right)-\left.\varphi_{t}^{\prime}(t, v)\right|_{v=W_{t}+C(t)}}{\sigma\left(t, \varphi\left(t, W_{t}+C(t)\right)\right)}, \quad C(0)=0 .
$$

The derivative $\varphi_{t}^{\prime}(t, v)$ in equation (7) can be found differentiating (6) by Leibniz rule:

$$
\varphi_{t}^{\prime}(t, v)=\sigma(t, \varphi(t, v)) \int_{\xi_{0}}^{\varphi(t, v)} \frac{\sigma_{t}^{\prime}(t, \psi) d \psi}{(\sigma(t, \psi))^{2}} .
$$

In what follows we call the function $\varphi(t, v)$ the structure of solution, and the function $C(t)$ is called drift function of equation (4).

\section{COMPARISON THEOREMS}

We return back to studying equations (1), (2). We let

$$
m(t)=\min _{s \leqslant t} W_{s}, \quad M(t)=\max _{s \leqslant t} W_{s}, \quad \operatorname{ran}(t, \varphi)=\{y \in R \mid y=\varphi(t, v), v \in R\} .
$$

We denote by $\varphi_{1}(t, v), C_{1}(t), G_{1}(t, u), \varphi_{2}(t, v), C_{2}(t), G_{2}(t, u)$ the structures of solutions, the drift functions and the functions $G(t, u)$ for equations (1), (2), respectively. Hereafter we assume that for all $t \geqslant 0$ the following conditions hold:

- For all $u \in \mathbb{R}$, the functions $\sigma_{i}(t, u),\left(\sigma_{i}\right)_{t}^{\prime}(t, u),\left(\sigma_{i}\right)_{u}^{\prime}(t, u), b_{i}(t, u)$ are continuous and the functions $\sigma_{i}^{-1}(t, u)$ are locally summable $i=1,2$.

- For all $u, u^{\prime} \in \mathbb{R}$ the inequality holds $\left|\sigma_{i}(t, u)-\sigma_{i}\left(t, u^{\prime}\right)\right| \leqslant K\left|u-u^{\prime}\right|$, where $K=$ const $>0$, $i=1,2$.

- For all $u \in \mathbb{R}$, the inequality

$$
\left|b_{i}(t, u)\right|+\left|\sigma_{i}(t, u)\right| \leqslant K(1+|u|)
$$

holds, where $K=$ const $>0, i=1,2$. 
We write equations (7) for $C_{i}(t), i=1,2$ :

$$
\frac{d C_{i}(t)}{d t}=\frac{b_{i}\left(t, \varphi_{i}\left(t, W_{t}+C_{i}(t)\right)\right)-\left.\left(\varphi_{i}\right)_{t}^{\prime}(t, v)\right|_{v=W_{t}+C_{i}(t)}}{\sigma_{i}\left(t, \varphi_{i}\left(t, W_{t}+C_{i}(t)\right)\right)}, \quad C_{i}(0)=0 .
$$

We denote the right hand sides of equations (8) by

$$
F_{i}(t, y)=\frac{b_{i}\left(t, \varphi_{i}\left(t, W_{t}+y\right)\right)-\left.\left(\varphi_{i}\right)_{t}^{\prime}(t, v)\right|_{v=W_{t}+y}}{\sigma_{i}\left(t, \varphi_{i}\left(t, W_{t}+y\right)\right)} .
$$

Lemma 1. Let the functions $F_{i}(t, y), i=1,2$, be continuous and for all $t \geqslant 0$ they satisfy the conditions:

(a) $\left|F_{i}(t, x)-F_{i}\left(t, x^{\prime}\right)\right| \leqslant L\left|x-x^{\prime}\right|, L=$ const $>0$, for all $x, x^{\prime} \in \mathbb{R}$ for a.e. $\omega$,

(b) $F_{2}(t, y) \geqslant F_{1}(t, y)$ for all $y \in \mathbb{R}$ for a.e. $\omega$.

Then $C_{2}(t) \geqslant C_{1}(t)$ for all $t \in I$ a.s., where $I$ is the common interval of existence of solutions to equations (8).

Proof. Assume that there exists a time $t_{1} \in I$, at which

$$
C_{2}\left(t_{1}\right)<C_{1}\left(t_{1}\right) \quad \text { a.s. }
$$

Since there exist solutions to equations (8) on the interval $I$ and $C_{2}(0)=C_{1}(0)$, there exists a time $t_{2}<t_{1}$ such that

$$
t_{2}=\sup \left\{t \in\left[0, t_{1}\right] \mid C_{2}(t) \geqslant C_{1}(t) \text { a.s. }\right\} \text {. }
$$

It follows from the definition of $t_{2}$ that $C_{2}\left(t_{2}\right)=C_{1}\left(t_{2}\right)$ and $C_{2}(t)<C_{1}(t)$ for each $t \in\left(t_{2}, t_{1}\right]$ with probability 1 . We denote $\delta(t)=C_{1}(t)-C_{2}(t), \delta(t) \geqslant 0$ for all $t \in\left[t_{2}, t_{1}\right]$ for a.e. $\omega$. On the other hand, since $\delta_{t}^{\prime}(t)=\left(C_{1}\right)_{t}^{\prime}(t)-\left(C_{2}\right)_{t}^{\prime}(t)$, then with probability 1 it holds that

$$
\begin{aligned}
0 & \leqslant \delta(t)=C_{1}\left(t_{2}\right)-C_{2}\left(t_{2}\right)+\int_{t_{2}}^{t}\left(F_{1}\left(s, C_{1}(s)\right)-F_{2}\left(s, C_{2}(s)\right)\right) d s \leqslant|(b)| \\
& \leqslant \int_{t_{2}}^{t}\left|F_{2}\left(s, C_{1}(s)\right)-F_{2}\left(s, C_{2}(s)\right)\right| d s \leqslant|(a)| \leqslant L \int_{t_{2}}^{t}\left|C_{1}(s)-C_{2}(s)\right| d s=L \int_{t_{2}}^{t} \delta(s) d s .
\end{aligned}
$$

By Grönwall's lemma, the latter relations mean that $\delta(t)=0$ for all $t \in\left[t_{2}, t_{1}\right]$ for a.e. $\omega$ and this contradicts (9). Therefore, $C_{2}(t) \geqslant C_{1}(t)$ for all $t \in I$ a.s.

Theorem 2. Assume that for all $t \geqslant 0$ the inequality holds:

$$
\sigma_{2}(t, u) \geqslant \sigma_{1}(t, u) \quad \text { for all } u \in \mathbb{R} .
$$

Then the following statements are true:

1. If $\varphi_{2}\left(t, m(t)+C_{2}(t)\right)>\varphi_{1}\left(t, m(t)+C_{1}(t)\right)$ for all $t \geqslant 0$ with probability 1 , then $\xi_{t}^{(2)}>\xi_{t}^{(1)}$ for all $t \geqslant 0$ a.s.

2. If $\varphi_{2}\left(t, m(t)+C_{2}(t)\right) \geqslant \varphi_{1}\left(t, m(t)+C_{1}(t)\right)$ for all $t \geqslant 0$ with probability 1 , then $\xi_{t}^{(2)} \geqslant \xi_{t}^{(1)}$ for all $t \geqslant 0$ a.s.

Proof. 1. According Theorem 1, for all fixed $t \geqslant 0$ the functions $\varphi_{2}\left(t, v+C_{2}(t)\right), \varphi_{1}\left(t, v+C_{1}(t)\right)$, $v \in[m(t), M(t)]$, solve the Cauchy problems

$$
\begin{aligned}
& \frac{d}{d v} \varphi_{i}\left(t, v+C_{i}(t)\right)=\sigma_{i}\left(t, \varphi_{i}\left(t, v+C_{i}(t)\right)\right), \\
& \left.\varphi_{i}\left(t, v+C_{i}(t)\right)\right|_{v=m(t)}=\varphi_{i}\left(m(t)+C_{i}(t)\right),
\end{aligned}
$$

with probability 1 , where $i=1,2$. 
In view of assumptions of Theorem 2, for these differential equations the comparison theorem for ODE holds [14, Sect. 2, Thm. 4] and therefore, $\varphi_{2}\left(t, v+C_{2}(t)\right)>\varphi_{1}\left(t, v+C_{1}(t)\right)$ for all $t \geqslant 0, v \in[m(t), M(t)]$ for a.e. $\omega$, and hence, $\xi_{t}^{(2)}>\xi_{t}^{(1)}$ for all $t \geqslant 0$ a.s.

2. Proof is similar to that of Lemma 1 .

Corollary 1. Assume that for all $t \geqslant 0$ the following conditions hold:

(a) $\varphi_{2}(t, v)>\varphi_{1}(t, v)$ for all $v \in\left[m(t)+C_{1}(t), M(t)+C_{2}(t)\right]$,

(b) $\sigma_{2}(t, u) \geqslant \sigma_{1}(t, u), \sigma_{2}(t, u)>0$ for all $u \in \mathbb{R}$,

(c) $C_{2}(t) \geqslant C_{1}(t)$ a.s.

Then Statement 1 of Theorem 2 holds.

Proof. According Condition (b) of Corollary 1, the function $\varphi_{2}(t, v)$ increases in $v$ for all $t \geqslant 0$ and hence, $\varphi_{2}\left(t, v_{2}\right) \geqslant \varphi_{2}\left(t, v_{1}\right)>\varphi_{1}\left(t, v_{1}\right)$ for all $v_{2} \geqslant v_{1}$. Thus, in order to check the assumptions of Theorem 2, it is sufficient to show that $m(t)+C_{2}(t) \geqslant m(t)+C_{1}(t)$ for all $t \geqslant 0$ with probability 1 . The latter inequality follows Condition $(c)$ of Corollary 1 .

Corollary 2. Statement 1 of Theorem 2 holds if for all $t \geqslant 0$

(a) $m(t)+C_{1}(t)>0$ with probability 1 ,

(b) $\sigma_{2}(t, u) \geqslant \sigma_{1}(t, u)>0$ for all $u \in \mathbb{R}$,

(c) $C_{2}(t) \geqslant C_{1}(t)$ with probability 1 ,

(d) $\xi_{0}^{(2)} \geqslant \xi_{0}^{(1)}>0$.

Proof. Since according Condition $(b)$ of Corollary 2 the function $\varphi_{2}(t, v)$ is increasing in $v$ for all $t \geqslant 0$, then $\varphi_{2}\left(t, m(t)+C_{2}(t)\right) \geqslant \varphi_{2}\left(t, m(t)+C_{1}(t)\right)$ for all $t \geqslant 0$ a.s. To complete the proof of Corollary 2, it remains to check Condition 1 of Theorem 2, By (6) and Condition (a) of Corollary 2, the relations

$$
G_{2}\left(t, \varphi_{2}\left(t, m(t)+C_{1}(t)\right)\right)=G_{1}\left(t, \varphi_{1}\left(t, m(t)+C_{1}(t)\right)\right)=m(t)+C_{1}(t)>0
$$

hold. Hence, $\varphi_{2}\left(t, m(t)+C_{1}(t)\right)>\varphi_{1}\left(t, m(t)+C_{1}(t)\right) \geqslant 0$ for all $t \geqslant 0$ a.s., therefore,

$$
\varphi_{2}\left(t, m(t)+C_{2}(t)\right)>\varphi_{1}\left(t, m(t)+C_{1}(t)\right)
$$

for almost all $\omega$.

Remark 1. One can show that if we replace the strict inequality in Conditions (a) of Corollaries 1, 2 by a non-strict one, the same arguing allows one to prove Statement 2 of Theorem 2 .

Theorem 3. Assume that for all $t \geqslant 0$ the following conditions hold:

(a) $\varphi_{2}\left(t, G_{1}(t, u)\right) \geqslant u$ for all $v \in \mathbb{R}$,

(b) $\sigma_{2}(t, u)>0$ for all $u \in \operatorname{ran}\left(t, \varphi_{2}\right)$,

(c) $C_{2}(t) \geqslant C_{1}(t)$ for almost all $\omega$.

Then $\xi_{t}^{(2)} \geqslant \xi_{t}^{(1)}$ for all $t \geqslant 0$ a.s.

Proof. By relation (6), the structure of solution to equation (2) is represented as a function of the structure of solution to equation (1):

$$
\varphi_{2}\left(t, u+C_{2}(t)\right)=\varphi_{2}\left(t, G_{1}\left(t, \varphi_{1}\left(t, u+C_{1}(t)\right)\right)+C_{2}(t)-C_{1}(t)\right) .
$$

In view of Condition (b) of Theorem 3 , the function $\varphi_{2}(t, v)$ is increasing in $v$ for all $t \geqslant 0$, therefore,

$$
\varphi_{2}\left(t, G_{1}\left(t, \varphi_{1}\left(t, v+C_{1}(t)\right)\right)+C_{2}(t)-C_{1}(t)\right) \geqslant \varphi_{2}\left(t, G_{1}\left(t, \varphi\left(t, v+C_{1}(t)\right)\right)\right) .
$$

By Condition (a) of Theorem 3 the latter inequality implies that for each $t \geqslant 0$ for all $v \in \mathbb{R}$ the inequality

$$
\varphi_{2}\left(t, v+C_{2}(t)\right) \geqslant \varphi_{1}\left(t, v+C_{1}(t)\right)
$$

holds with probability 1 . Substituting $v=W_{t}$ into $(10)$, we obtain that $\xi_{t}^{(2)} \geqslant \xi_{t}^{(1)}$ for each $t \geqslant 0$ for almost all $\omega$. 
The proof of Theorem 4 given below is similar to the proof of Theorem 3 , just in this case due to Condition (b) of Theorem 4 the function $\varphi_{2}(t, v)$ decreases in $v$ for all $t \geqslant 0$.

Theorem 4. Assume that for all $t \geqslant 0$ the following inequalities hold:

(a) $\varphi_{2}\left(t, G_{1}(t, u)\right) \leqslant u$ for all $u \in \mathbb{R}$,

(b) $\sigma_{2}(t, u)<0$ for all $v \in \operatorname{ran}\left(t, \varphi_{2}\right)$,

(c) $C_{2}(t) \geqslant C_{1}(t)$ for almost all $\omega$.

Then $\xi_{t}^{(2)} \leqslant \xi_{t}^{(1)}$ for all $t \geqslant 0$ with probability 1 .

If the functions $\sigma_{i}(t, u)$ are also independent of the variable $t$, then the functions $G_{i}(u)=$ $G_{i}(t, u)$ are also independent of the variable $t$, where $i=1,2$.

Lemma 2. Let $\eta_{t}^{(i)}$ be solutions of $O D E$

$$
d \eta_{t}^{(i)}=\sigma_{i}\left(\eta_{t}^{(i)}\right) * d W_{t},\left.\quad \eta_{t}^{(i)}\right|_{t=0}=\eta_{0}^{(i)}, \quad i=1,2,
$$

and for each $u \in \mathbb{R}$ the conditions hold:

(a) $\sigma_{i}(u) \geqslant 0$, where $i=1,2$,

(b) $\varphi_{2}\left(G_{1}(u)\right) \geqslant u$, where $\varphi_{2}(v)$ is the structure of solutions to equation for $\eta_{t}^{(2)}$ for all $v \in \mathbb{R}$.

Then for each $u \in \mathbb{R}$ the inequality holds:

$$
G_{1}(u) \geqslant G_{2}(u) \text {. }
$$

Proof. Taking into consideration that the coefficients of the equations (11) and the structures of their solutions are independent of the time, we write relation (6) for $\varphi_{2}(v)$ :

$$
G_{2}\left(\varphi_{2}(v)\right)=v+C(t)
$$

We observe that $C^{\prime}(t)=0$ for all $t \geqslant 0$ by (7), therefore, $C(t)=0$ for all $t \geqslant 0$ since $C(0)=0$. Under assumptions of Lemma 2 , the functions $G_{i}(u), i=1,2$, are non-decreasing. We substitute $v=G_{1}(u)$ into 13 and by Condition (b) of Lemma 2 we obtain:

$$
G_{1}(u)=G_{2}\left(\varphi_{2}\left(G_{1}(u)\right)\right) \geqslant G_{2}(u) .
$$

Remark 2. The results presented in work [8] follow inequality (12). Thus, Lemma 2 says that the results obtained by O'Brien in work [8] are particular case of our results.

Example 1. Let us provide an example of application of Theorem 3. We consider two SDEs with Stratonovich integrals, whose diffusion coefficients are different:

$$
\begin{aligned}
& d \xi_{t}^{(1)}=1 * d W_{t}+2 d t, \quad \xi_{0}^{(1)}=0, \\
& d \xi_{t}^{(2)}=2 \sqrt{\xi_{t}^{(2)}-1} * d W_{t}+4 \sqrt{\xi_{t}^{(2)}-1} d t, \quad \xi_{0}^{(2)}=1 .
\end{aligned}
$$

Since these equations satisfy the assumptions of Theorem 3 , we have $\xi_{t}^{(2)} \geqslant \xi_{t}^{(1)}$ for each $t \geqslant 0$ with probability 1 . The latter inequality can be checked directly since the solutions to the considered equations are of form $\xi_{t}^{(1)}=W_{t}+2 t, \xi_{t}^{(2)}=\left(W_{t}+2 t\right)^{2}+1$.

\section{Stability With probability 1}

We proceed to studying the stability with probability 1 for equation (3). The main idea of our approach is to apply the comparison theorems for estimating the perturbed solution to equation (3) by means of solution to SDE stable with probability 1 . It was shown in work [1] that as such equation, the equation

$$
d \eta_{t}^{(\alpha)}=t \eta_{t}^{(\alpha)} * d W_{t}-t^{\alpha+\frac{1}{2}} \eta_{t}^{(\alpha)} d t, \quad t \geqslant 0,
$$

can serve. Its perturbed solution is stable with probability 1 for all $\alpha>0$. 
We denote $\operatorname{sgn}(x)=-1$ as $x<0, \operatorname{sgn}(x)=0$ as $x=0, \operatorname{sgn}(x)=1$ as $x>0$. Let $\varphi_{1}(t, v), C_{1}(t), G_{1}(t, u), \varphi_{2}(t, v), C_{2}(t), G_{2}(t, u)$ be the structures of solutions, drift functions and functions $G(t, u)$ for equations (3), (14), respectively.

Theorem 5. Assume that for all $t>0$ the following conditions hold:

(a) $C_{2}(t) \geqslant C_{1}(t)$ for almost all $\omega$,

(b) $\operatorname{sgn}(\sigma(t, u))=\operatorname{sgn}(u)$ for all $u \neq 0, u \in \mathbb{R}$,

(c) $\sigma(t, u) \leqslant t u$ for all $u \neq 0, u \in \mathbb{R}$.

Then the perturbed solution to equation (3) is stable with probability 1.

Proof. It is sufficient to show that for all $t \geqslant 0$ the inequality $\left|\xi_{t}\right| \leqslant K \eta_{t}^{(\alpha)}$ holds with probability 1 (see [1]), where $\eta_{t}^{(\alpha)}$ is the perturbed solution of equation (14) stable with probability 1 . The structure of solution to equation $(14)$ is of form

$$
\varphi_{2}\left(t, v+C_{2}(t)\right)=\eta_{0} \exp \left\{t\left(v+C_{2}(t)\right)\right\} .
$$

Without loss of generality we let $\eta_{0}>0$ since otherwise we can make the change $\widetilde{\eta_{0}}=-\eta_{0}>0$.

Let us show first that $\xi_{t} \leqslant K \eta_{t}^{(\alpha)}$ for all $t \geqslant 0$ with probability 1 . In order to do this, we apply Theorem 3 to equations (3), (14). Since $\varphi_{2}(t, v)>0$, we have $\sigma_{2}(t, u)=t u>0$ for all $u \in \operatorname{ran}\left(t, \varphi_{2}\right)$, that is, Condition (b) of Theorem 3 is satisfied. Condition (c) of Theorem 3 is true due to Condition (a) of Theorem 5. It remains to check Condition (a) of Theorem 3 .

$$
K \eta_{0} \exp \left\{t G_{1}(t, u)\right\} \geqslant u \text {. }
$$

We divide both sides by $K \eta_{0}$ and calculate the logarithm:

$$
t G_{1}(t, u) \geqslant \ln \left(\frac{u}{K \eta_{0}}\right) .
$$

Since $K \eta_{0} \exp \left\{t G_{1}(t, 0)\right\} \geqslant 0$, to check the latter inequality, it is sufficient to show that

$$
\frac{d}{d u}\left(t G_{1}(t, u)-\ln \left(\frac{u}{\eta_{0}}\right)\right) \geqslant 0
$$

that is,

$$
\frac{t}{\sigma(t, u)} \geqslant \frac{1}{u}
$$

The latter inequality is true thanks to Conditions (b), (c) of Theorem 5 .

Arguing as above and applying Theorem 4, we see that Conditions (a)-(c) of Theorem 5 are sufficient to ensure the inequality $\xi_{t} \geqslant-K \eta_{t}^{(\alpha)}$ for all $t \geqslant 0$ with probability 1.

Remark 3. In the case $\sigma(t, u)>0$ for all $t \geqslant 0, \in R$, Conditions (b), (c) of Theorem 5 can be replaced by Condition $\sigma(t, u) \leqslant t u$ for all $u \geqslant 0$.

Remark 4. Since $C_{2}(t)=\int_{0}^{t}\left(s^{(\alpha+1) / 2}+W_{s}\right) d s / t$, Condition (a) of Theorem 5 is reduced to checking the inequality $t C_{1}(t) \leqslant \int_{0}^{t}\left(s^{(\alpha+1) / 2}+W_{s}\right) d s$.

Example 2. Let us provide an example of application of Theorem 5. We consider SDE with Stratonovich integral:

$$
d \xi_{t}=\sigma\left(t, \xi_{t}\right) * d W_{t}-t^{\alpha+\frac{1}{2}} \xi_{t} d t, \quad t \geqslant 0
$$

where $\sigma(t, u)=t u / 2$ as $u>0, \sigma(t, u)=0$ as $u=0, \sigma(t, u)=2 t u$ as $u>0$.

The function $\sigma(t, u)$ satisfies the Lipschitz condition. Let us show that for equation (16) all conditions of Theorem 5 are satisfied. Conditions (b), (c) of Theorem 5 hold by the definition of the function $\sigma(t, u)$. Condition (a) of Theorem 5 can be checked by straightforward substitution. Hence, the perturbed solution to equation (16) is stable with probability 1. 


\section{BIBLIOGRAPHY}

1. A.S. Asylgareev, F.S. Nasyrov. Theorems of comparison and stability with probability 1 for onedimensional stochastic differential equations // Sibir. Matem. Zhurn. 57:5, 969-977 (2016). [Siber. Math. J. 57:5, 754-761 (2016).]

2. A.V. Skorokhod. Studies in the theory of random processes. Izd. Kiev. Univ., Kiev (1961). [AddisonWesley Publish, Reading, Mass. (1965).]

3. N. Ikeda, Sh. Watanabe. Stochastic differential equations and diffusion processes. North-Holland Publishing Company, Amsterdam (1981).

4. T. Yamada. On a comparison theorem for solutions of stochastic differential equations and its applications // J. Math. Kyoto Univ. 13:3, 497-512 (1973).

5. C. Geib, R. Manthey. Comparison theorems for stochastic differential equations in finite and infinite dimensions // Stoch. Proc. Appl. 53:1, 23-35 (1994).

6. S. Kotelenez. Comparison methods for a class of function-valued stochastic partial differential equations // Probab. Theor. Relat. Fields. 93:1, 1-19 (1992).

7. P. Peng, X. Zhu. Necessary and sufficient condition for comparison theorem of 1-dimensional stochastic differential equations // Stoch. Proc. Appl. 116:3, 370-380 (2006).

8. G.L. O'Brien. A new comparison theorem for solutions of stochastic differential equations // Stoch. 13:3, 497-512 (1973).

9. R. Z. Khas'minskiī. Stability of systems of differential equations under random perturbations of their parameters. Nauka, Moscow (1969). [Stochastic stability of differential equations. Sijthoff \& Noordhoff, Alphen aan den Rijn (1980).]

10. H.J. Kushner. Stochastic stability and control. Academic Press, New York (1969).

11. X. Mao. Exponential stability ofs tochastic differential equations. Marcel Dekker, New York (1994).

12. F.S. Nasyrov. Local times, symmetric integrals and stochastic analysis. Fizmatlit, Moscow (2011). (in Russian).

13. A.S. Asylgareev. On comparison of solutions to homogeneous stochastic differential equations // Proc. Inter. Youth Sci. Forum "Lomonosov-2017". Maks Press, Moscow (2017). (in Russian).

14. A.B. Vasileva, N.N. Nefedov. Comparison theorems. Chaplygin method of differential inequalities. Preprint, Moscow State Univ., Moscow (2007). (in Russian).

Arthur Salavatovich Asylgareev,

Ufa State Aviation Technical University,

Karl Marx str. 12,

450000, Ufa, Russia

E-mail: asylgareevarthur@gmail.com 